\title{
Prognostic factors for severe Pneumocystis jiroveci pneumonia of non-HIV patients in intensive care unit: a bicentric retrospective study
}

Li Weng $^{1 \dagger}$, Xu Huang ${ }^{2 \dagger}$, Lie Chen ${ }^{3}$, Li-Qin Feng ${ }^{4}$, Wei Jiang ${ }^{1}$, Xiao-Yun Hu ${ }^{1}$, Jin-Min Peng ${ }^{1}$, Chun-Yao Wang ${ }^{1}$, Qing-Yuan Zhan $^{2+}$ and Bin Du ${ }^{1 *+}$

\begin{abstract}
Background: Pneumocystis jiroveci pneumonia (PJP) in non-HIV patients is still a challenge for intensivists. The aim of our study was to evaluate mortality predictors of PJP patients requiring Intensive care unit (ICU) admission.

Methods: Retrospectively review medical records of patients with diagnosis of PJP admitted to four ICUs of two academic medical centers from October 2012 to October 2015.

Results: Eighty-two patients were enrolled in the study. Overall hospital mortality was $75.6 \%$. Compared with survivors, the non-survivors had older age (55 \pm 16 vs. $45 \pm 17, p=0.014)$, higher APACHE II score (20 \pm 5 vs. $17 \pm 5$, $p=0.01)$, lower white blood cell count (7.68 \pm 3.44 vs. $10.48 \pm 4.62, p=0.005)$, less fever $(80.6 \%$ vs. $100 \%, p=0.033)$, more hypotension ( $58.1 \%$ vs. $20 \%, p=0.003$ ), more pneumomediastinum ( $29 \%$ vs. $5 \%, p=0.027$ ). Logistic regression analysis demonstrated that age [odds ratio $(\mathrm{OR}) 1.051 ; 95 \% \mathrm{Cl} 1.007-1.097 ; p=0.022$, white blood cell count [OR 0.802; $95 \% \mathrm{Cl} 0.670-0.960 ; p=0.016]$, and pneumomediastinum [OR 16.514; $95 \% \mathrm{Cl} 1.330-205.027 ; p=0$. 029] were independently associated with hospital mortality.
\end{abstract}

Conclusions: Mortality rate for non-HIV PJP patients requiring ICU admission was still high. Poor prognostic factors included age, white blood cell count and pneumomediastinum.

Keywords: Pneumocystis pneumonia, Intensive care units, Mortality

\section{Background}

With the widespread use of Pneumocystis jirovecii pneumonia (PJP) prophylaxis and highly active antiretroviral therapy (HAART), the incidence and mortality of PJP in HIV patients have declined substantially in Europe and the United States [1, 2]. Current estimates of hospital mortality for PJP in HIV patients range from $7 \%$ to $11 \%$ [3]. Most importantly, respiratory failure due to PJP requiring ICU admission was less common in HIV patients [4]. In contrast, PJP rate is increasing in nonHIV patient [5] and the reported mortality of PJP in

\footnotetext{
*Correspondence: dubin98@gmail.com

${ }^{\dagger}$ Equal contributors

${ }^{1}$ Medical ICU, Peking Union Medical College Hospital, Peking Union Medical College and Chinese Academy of Medical Sciences, 1 Shuaifuyuan,

Dongcheng district, Beijing 100730, China

Full list of author information is available at the end of the article
}

immunocompromised non-HIV patients ranges from $48 \%$ to $67 \%$ [3]. The clinical course, and inflammatory response might contribute to the different mortality rates between the two groups. In addition, the sample sizes of previous studies exploring the prognostic factor were small [6-8]. The prognostic factor for severe PJP in the intensive care unit (ICU) setting has not been well described.

In order to determine the prognostic factors for PJP in ICU setting, we retrospectively collected data for a consecutive series of PJP patients requiring ICU admission from October 2012 to October 2015.

\section{Methods}

\section{Study design}

We conducted a bicentric retrospective cohort study in 4 ICUs at 2 academic medical centers, including a 
medical ICU, an emergency ICU, and a general ICU at Peking Union Medical College Hospital (PUMCH 1800 beds), and a medical ICU at China-Japan Friendship Hospital (CJFH 1610 beds). All four ICUs contain 83 beds during the study period.

All patients discharged with diagnosis of PJP from October 2012 to October 2015 were screened for eligibility. Inclusion criteria: (1) PJP, confirmed by Polymerase Chain Reaction (PCR) or methenamine silver stain of samples from bronchoalveolar lavage fluid (BALF), aspirate or sputum; (2) HIV tests negative; (3) ICU admission during index hospitalization due to respiratory insufficiency.

Patients without immunocompromised background, less than 18 years old or pregnant were excluded.

\section{Data collection}

Data were gathered retrospectively from medical records at both PUMCH and CJFH by the investigators (L.C., L-Q.F., L.W., and X.H.). Patient identifiers were removed from the final data sheet and were coded with a numbered assignment.

Demographic, laboratory, and clinical data were collected, including age, gender, microbiological findings for PJP, severity of illness based on the acute physiology and chronic health evaluation (APACHE) II score, comorbidities, complications, the time of PJP symptom onset, the time of PJP diagnosis, the time to appropriate antibiotics administration, and the chest radiographs and CT scan findings. Data on mechanical ventilation included type of oxygen therapy or ventilatory support on ICU admission; tidal volume, plateau pressure, positive end-expiratory pressure (PEEP), fraction of inspired oxygen $(\mathrm{FiO} 2)$ and recruitment maneuver on ICU admission; maximal tidal volume, maximal PEEP during ICU stay. Hospital mortality was the primary outcome of our study.

\section{Definition}

(1) PJP was defined as: symptoms and radiographs compatible with PJP [9]; confirmed by PCR or methenamine silver stain of samples from bronchoalveolar lavage fluid (BALF), aspirate or sputum. (2) ventilator-associated pneumonia (VAP) was defined as a new lung parenchymal opacity on a chest radiograph of a patient intubated for more than $48 \mathrm{~h}$; and simultaneous presentation of two or more of: purulent secretion from tracheal; temperature of less than $36^{\circ} \mathrm{C}$ or more than $38^{\circ} \mathrm{C}$; white blood cell count (WBC) of less than $4 \times 109 / \mathrm{L}$ or more than $10 \times 109 / \mathrm{L}$. (3) Pulmonary aspergillosis was defined by one host factor criterion, one microbiological criterion and one major clinical criterion (or 2 minor criteria) according to an international consensus from experts of the European Organization for Research and Treatment of Cancer
(EORTC) and the National Institute of Allergy and Infectious Diseases Mycoses Study Group [10]. (4) Cytomegalovirus (CMV) infection was confirmed if pp65-antigenemia assay or CMV DNA assay were positive [11]. (5) Barotrauma was defined as pneumomediastinum or pneumothorax. (6) Pneumomediastinum was diagnosed as the presence of free air in the mediastinal cavity by CT scan or Chest x-ray showing hyperlucent lines outlining the lateral heart borders with subcutaneous emphysema around neck and chest region.

This study was approved by the institutional review board of Peking Union Medical College Hospital (S-K116). Due to the retrospective nature of the study, informed written consent was waived.

\section{Statistical analysis}

For the statistical analysis, continuous data were compared with use of the Student's $t$ test or Mann-Whitney test as appropriate. Statistical analysis of non-continuous dichotomous data was compared by the chi-square test or the Fisher's Exact Test as appropriate. Logistic regression models were used to determine the effect of prognostic factors on hospital death by means of stepwise backward elimination procedures, after adjusting for covariates of which the $\mathrm{p}$ values were less than 0.1. All statistical analyses were performed using SPSS statistical software (version 22.0; IBM Inc., Armonk, NY). All p values were two sided, and statistical significances were accepted for $\mathrm{p}<0.05$.

\section{Results}

Overall, 348 patients were discharged with a diagnosis of PJP during the study period, of whom 266 met exclusion criteria. Consequently, 82 patients were available for the final analysis, including 72 patients $\mathrm{PUMCH}$ and 10 patients in CJFH (Fig. 1).

Table 1 shows characteristics and outcomes of confirmed PJP in non-HIV patients. The entire 82 patient cohort had a mean age $53 \pm 17$ years of and APACHE II score of $19 \pm 5$, with $41.4 \%$ male. Most of the patients had an underlying disease of connective tissue disease (79.3\%) and history of corticosteroid therapy (84.1\%). All patients were treated with trimethoprim-sulfamethoxazole (TMPSMX). Not a single patient received PJP prophylaxis. PJP was diagnosed by methenamine silver stain, PCR, or both in 13,45 , and 24 patients respectively, without any difference between 2 hospitals (Additional file 1: Table S2). PJPPCR positive samples included sputum $(n=5)$, trachea aspirate $(n=17)$, and BALF $(n=49)$. Methenamine silver stain positive samples included sputum $(n=4)$, trachea aspirate $(n=12)$, and BALF $(n=19)$. All pulmonary aspergillosis was diagnosed as EORTC probable invasive aspergillosis with the presence of a host factor, a clinical criterion, and a mycological criterion. For the 38 patients 
348 patients discharged with diagnosis of Pneumocystis jiroveci Pneumonia screened for eligibility

Excluded

- HIV 7 patients

- PCR negative and Methenamine silver stain negative 35 patients

- Without ICU admission 224 patients

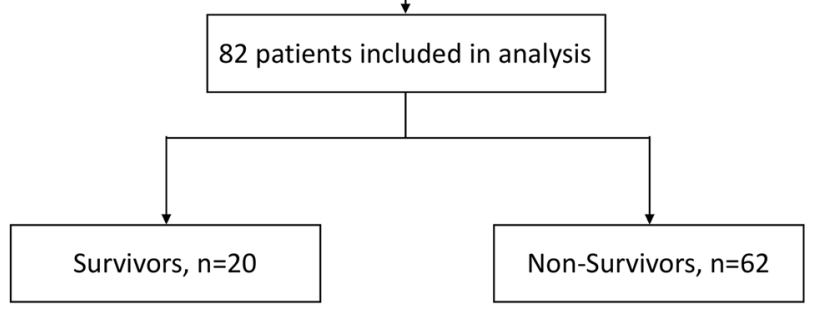

Fig. 1 The patient flowchart with respect to inclusion and exclusion

receiving caspofungin, total days on caspofungin were $8 \pm$ 6. Twenty-five patients received empirical caspofungin therapy for less than 7 days for suspected invasive fungal infection. Another 6 patients received caspofungin as a combination therapy with amphotericin $B$ or voriconazole for aspergillosis. Although the clinical efficacy of caspofungin as salvage therapy for PJP remained controversial [12], it was administered in 7 patients in our cohort study as a salvage regimen of whom 5 patients died during study period.

During their hospital stay, 62 (75.6\%) of the 82 patients died. Compared with survivors, the nonsurvivors had older age ( $55 \pm 16$ vs. $45 \pm 17, p=0.014$ ), higher APACHE II score ( $20 \pm 5$ vs. $17 \pm 5, p=0.01$ ), lower WBC (7.68 \pm 3.44 vs. $10.48 \pm 4.62, p=0.005)$, less fever $(80.6 \%$ vs. $100 \%, p=0.033)$, more hypotension (58.1\% vs. $20 \%, p=0.003$ ), and more pneumomediastinum ( $29 \%$ vs. $5 \%, p=0.027)$, while the difference was not statistically significant for lymphocyte counts, CD4 cell count, type of respiratory support on ICU admission. Four patients received high-frequency oscillatory ventilation and one patient received extracorporeal membrane oxygenation. All these five patients died during the hospital stay.

To investigate the role of potential confounding prognostic factors, a multivariate analysis was performed for hospital mortality (Table 2). The Hosmer and Lemeshow goodness-of-fit test were not rejected $(p=0.640)$, indicating adequate model fit. No interaction terms were found to be significant in this model, and there was no collinearity between any of the independent variables. The multivariate model indicated that age [odds ratio (OR)1.051; $95 \%$ CI 1.007-1.097; $p=0.022$ ], WBC [OR 0.802; $95 \%$ CI $0.670-0.960 ; p=0.016]$, and pneumomediastinum [OR
16.514; 95 \% CI 1.330-205.027; $p=0.029$ ] were independently significantly associated with hospital mortality.

CT scans were performed for all those 82 patients on ICU admission. Pneumomediastinum was confirmed by $\mathrm{CT}$ in 14 patients and chest $\mathrm{x}$-ray plus subcutaneous emphysema in 5 patients. Six patients developed pneumomediastinum before hospital admission. Other 13 patients had time intervals between ICU admission and pneumomediastinum with a mean of 9 days (range 2-30 days). The potential risk factors contributed to pneumomediastinum were listed in Table 3 . There was statistically significant difference in the percentage of patients treated with non-rebreathing mask (NRM) on ICU admission between pneumomediastinum and nonpneumomediastinum (47.4 \% vs. $23.8 \%, p=0.048)$. Tidal volume, plateau pressure, and PEEP was similar between those two groups.

\section{Discussion}

In this bicentric retrospective observational study across 4 Chinese ICUs, we found that age, WBC, and pneumomediastinum were significantly associated with hospital mortality in non-HIV immunocompromised patients with severe PJP who had been admitted to ICU. Use of non-rebreathing mask might contribute to the development of pneumomediastinum.

The most interesting finding of our study was that pneumomediastinum was associated with increased hospital mortality. Pneumomediastinum is the presence of extraalveolar air in the mediastinum, which is believed to arise from free air leaking from ruptured alveoli. It was described as an uncommon complication of opportunistic infections in HIV-infected patients [13, 14]. However, 24.4 \% patients in our study developed pneumomediastinum. The 
Table 1 Characteristics and outcomes of confirmed pneumocystis jiroveci pneumonia in non-HIV patients

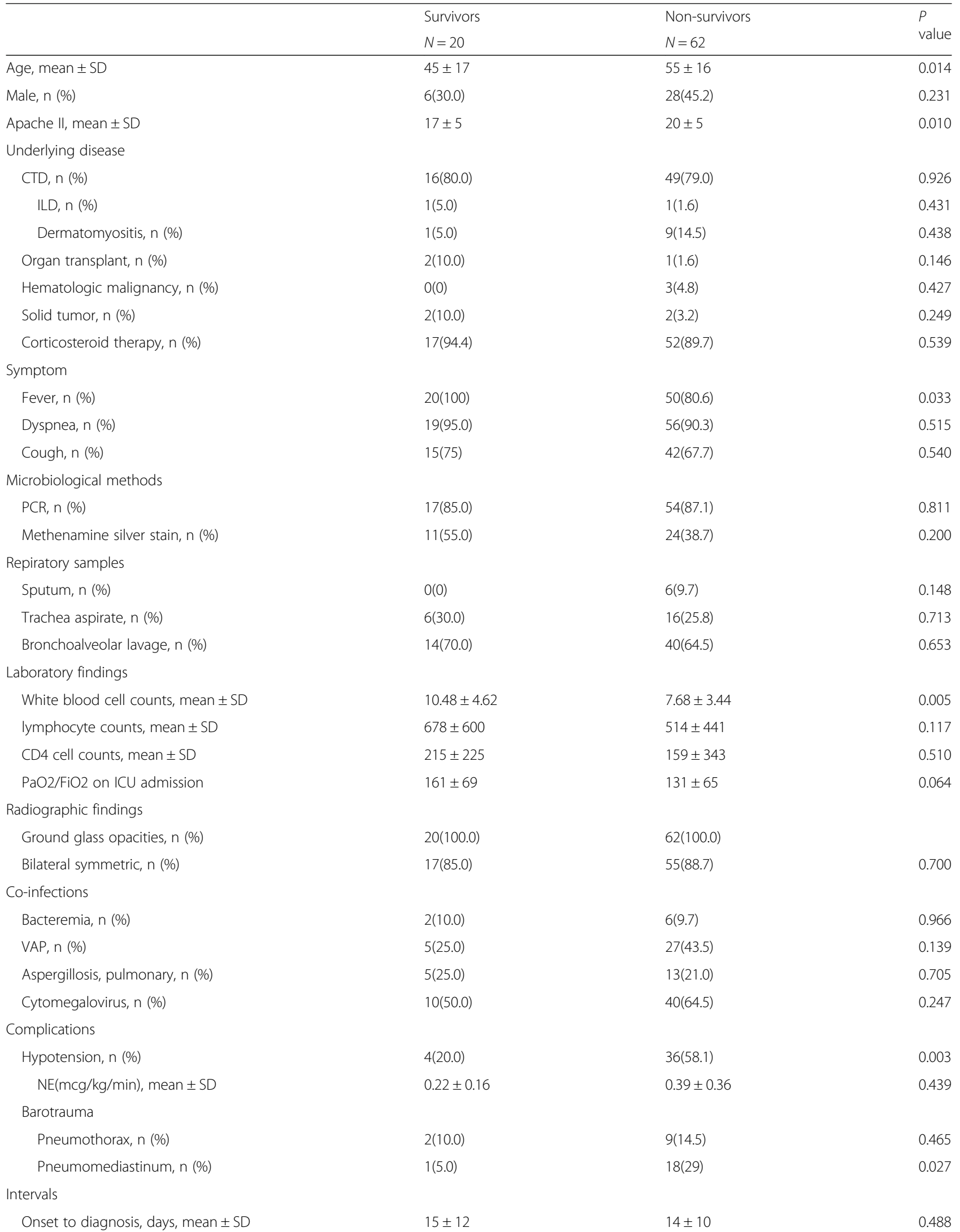


Table 1 Characteristics and outcomes of confirmed pneumocystis jiroveci pneumonia in non-HIV patients (Continued)

\begin{tabular}{|c|c|c|c|}
\hline Onset to intubation, days, mean \pm SD & $10 \pm 6$ & $12 \pm 11$ & 0.786 \\
\hline Onset to TMP/SMZ, days, mean \pm SD & $14 \pm 18$ & $11 \pm 10$ & 0.538 \\
\hline \multicolumn{4}{|l|}{ Respiratory support } \\
\hline IPPV during ICU stay, n (\%) & 16(80.0) & 59(95.2) & 0.057 \\
\hline NPPV on ICU admission, n (\%) & $4(20.0)$ & $8(12.9)$ & 0.474 \\
\hline IPPV on ICU admission, n (\%) & $10(50.0)$ & $36(58.1)$ & 0.527 \\
\hline NRM on ICU admission, n (\%) & $6(30.0)$ & 18(29.0) & 0.934 \\
\hline \multicolumn{4}{|l|}{ Medication } \\
\hline Adjunctive steroid, n (\%) & $16(80.0)$ & $48(77.4)$ & 0.808 \\
\hline Caspofungin, n (\%) & $7(35.0)$ & $31(50.0)$ & 0.242 \\
\hline
\end{tabular}

Values are expressed as the mean \pm SD or Number (\%), unless otherwise indicated. CTD Connective Tissue Disease, ILD Interstitial lung Disease, $P C R$ polymerase chain reaction, BALF bronchoalveolar lavage fluid, VAP ventilator-associated pneumonia, NE norepinephrine, TMP/SMZ trimethoprim-sulfamethoxazole, IPPV Invasive positive pressure ventilation, NPPV noninvasive positive pressure ventilation, NRM non-rebreathing mask

incidence rate discrepancies may be due to different underlying disease (HIV vs. Non-HIV) and few reported incidence rate in previous studies. A retrospective radiographic analysis reported an incidence rate of $11.1 \%$ (4 of 36) in a cohort of moderate non-HIV PJP patients with a mortality rate of $33.3 \%$ [15]. We also found more pneumomediastinum developed in the NRM group. This might be explained by higher trans-pulmonary pressure and tidal volume during spontaneous breath resulting in air leak, which was consistent with a previous report of HIV patients [13]. Delayed intubation was considered as a risk factor for worse outcome [6]. Although there was no difference in the time interval from symptom onset to intubation in our study, use of non-rebreathing mask instead of positive pressure support on ICU admission suggested delay intubation which was very difficult to define.

Development of pneumothorax was independently associated with increased mortality in previous studies $[6,16]$. We did not find any difference in mortality between patients with and without pneumothorax. Protective lung ventilation strategies might account for the different findings. The tidal volumes in our study were smaller than Festic and colleagues $(7 \mathrm{ml} / \mathrm{kg}$ vs. $10 \mathrm{ml} / \mathrm{kg})$. Despite the application of protective lung ventilation, one fourth patient in this cohort developed pneumomediastinum, which suggested that pneumomediastinum was not a complication of intervention. As Cho et al. [17] reported, the

Table 2 Multivariate analysis for predictors of death in patients with confirmed pneumocystis jiroveci pneumonia in non-HIV patients

\begin{tabular}{llll}
\hline & $\begin{array}{l}\text { Multivariate analysis } \\
\text { Odds Ratio }(95 \% \mathrm{Cl})\end{array}$ & Wald stat. & $P$ value \\
\hline Age & $1.051(1.007-1.097)$ & 5.238 & 0.022 \\
White blood cell counts & $0.802(0.670-0.960)$ & 5.787 & 0.016 \\
Pneumomediastinum & $16.514(1.330-205.027)$ & 4.761 & 0.029 \\
\hline
\end{tabular}

The risk factors removed from the logistic regression model including: APACHE II; Fever; PaO2/FiO2 on ICU admission; IPPV on ICU admission; Hypotension development of the pulmonary cysts and bronchiectasis that were noted in follow up CT but were not visible on $\mathrm{CT}$ at admission could be risk factors for development of pneumomediastinum.

We also found lower WBC was related to increased mortality. Although WBC has never been reported as risk factors, previous study [18] suggested a trend of higher WBC in non-HIV patients and survivors, which was consistent with our findings.

Overall mortality of the patients in our study was $75.6 \%$. Although the reported mortality rates of ICU nonHIV patients with PJP in previous studies were 38.9$84.2 \%[6-8,18-28]$ (Additional file 2: Table S1), most of the mortality rates were less than $70 \%$, which were lower than that of our study. The high hospital mortality rate in our study possibly was related to different underlying diseases and no prophylaxis of PJP for those patients. However, although prophylaxis for PJP was recommended for HIV patients, the efficacy of prophylaxis for immunocompromised non-HIV patients has not been well established [29-31], especially for the patients with underlying disease of connective tissue disease. In a recent study of ICU patients with PJP, adjunctive steroid was associated with increased mortality [16]. This might be the cause of high hospital mortality in our study. Considering that most of the patients in our study received steroid therapy before ICU admission, the use of steroid was not avoidable. Moreover, there was no difference in steroid therapy between survivors and non-survivors, and the effects of other covariates remained significant.

In a retrospective study, Chen and colleagues reported the characteristics and prognostic factors of 69 HIV-negative patients with PJP from PUCMH during 10 -year study period [25]. In comparison, we had enrolled 72 patients with confirmed PJP during a 3year period in our cohort (Additional file 1: Table S2, Additional file 3: Table S3). Increasing awareness of the disease in immunocompromised patients among 
Table 3 Risk factors for Pneumomediastinum of confirmed pneumocystis jiroveci pneumonia in non-HIV patients

\begin{tabular}{|c|c|c|c|}
\hline & \multirow{2}{*}{$\begin{array}{l}\text { Pneumomediastinum } \\
N=19\end{array}$} & \multirow{2}{*}{$\begin{array}{l}\text { Non-Peumomediastinum } \\
N=63\end{array}$} & \multirow[t]{2}{*}{$P$ value } \\
\hline & & & \\
\hline Age, mean $\pm S D$ & $51 \pm 19$ & $53 \pm 16$ & 0.606 \\
\hline Male, n (\%) & $8(42.1)$ & $26(41.3)$ & 0.948 \\
\hline Apache II, mean \pm SD & $19 \pm 5$ & $19 \pm 5$ & 0.948 \\
\hline \multicolumn{4}{|l|}{ Underlying conditions } \\
\hline CTD, n (\%) & $16(84.2)$ & 49(77.8) & 0.544 \\
\hline ILD, n (\%) & $1(5.3)$ & $1(1.6)$ & 0.412 \\
\hline Dermatomyositis, n (\%) & $1(5.3)$ & $9(14.3)$ & 0.440 \\
\hline Organ transplant, n (\%) & $1(5.3)$ & $2(3.2)$ & 0.552 \\
\hline Hematologic malignancy, n (\%) & $0(0)$ & $3(4.8)$ & 0.448 \\
\hline Solid tumor, n (\%) & $1(5.3)$ & $3(4.8)$ & 0.659 \\
\hline Corticosteroid therapy, n (\%) & 17(89.5) & $52(82.5)$ & 0.722 \\
\hline \multicolumn{4}{|l|}{ Symptom } \\
\hline Fever, n (\%) & 17(89.5) & $53(84.1)$ & 0.563 \\
\hline Dyspnea, n (\%) & 19(100) & $56(88.9)$ & 0.192 \\
\hline Cough, n (\%) & 14(73.7) & $43(68.3)$ & 0.652 \\
\hline \multicolumn{4}{|l|}{ Laboratory findings } \\
\hline White blood cell counts, mean \pm SD & $7.85 \pm 2.92$ & $8.52 \pm 4.18$ & 0.518 \\
\hline lymphocyte counts, mean \pm SD & $601 \pm 618$ & $540 \pm 444$ & 0.717 \\
\hline CD4 cell counts, mean \pm SD & $246 \pm 583$ & $156 \pm 186$ & 0.886 \\
\hline $\mathrm{PaO} 2 / \mathrm{FiO} 2$ on ICU admission & $144 \pm 53$ & $136 \pm 70$ & 0.663 \\
\hline \multicolumn{4}{|l|}{ Co-infections } \\
\hline Aspergillosis, pulmonary, n (\%) & $3(15.8)$ & 15(23.8) & 0.544 \\
\hline Cytomegalovirus, pulmonary, n (\%) & $12(63.2)$ & $38(60.3)$ & 0.824 \\
\hline \multicolumn{4}{|l|}{ Intervals } \\
\hline Onset to diagnosis, days, mean \pm SD & $13 \pm 5$ & $14 \pm 12$ & 0.480 \\
\hline Onset to intubation, days, mean \pm SD & $11 \pm 6$ & $12 \pm 11$ & 0.753 \\
\hline Onset to $T M P / S M Z$, days, mean \pm SD & $9 \pm 6$ & $12 \pm 13$ & 0.230 \\
\hline \multicolumn{4}{|l|}{ Respiratory support } \\
\hline IPPV during ICU stay, n (\%) & 18(94.7) & $57(90.5)$ & 0.560 \\
\hline $\mathrm{VT}$ maximal $(\mathrm{ml} / \mathrm{kg})$, mean $\pm \mathrm{SD}$ & $7.1 \pm 2.0$ & $8.1 \pm 2.4$ & 0.208 \\
\hline PEEP maximal $(\mathrm{cmH} 2 \mathrm{O})$, mean $\pm \mathrm{SD}$ & $11 \pm 5$ & $10 \pm 4$ & 0.415 \\
\hline NPPV on ICU admission, n (\%) & $3(15.8)$ & $9(14.3)$ & 0.871 \\
\hline IPAP $(\mathrm{cmH} 2 \mathrm{O})$, mean $\pm \mathrm{SD}$ & $14 \pm 3$ & $11 \pm 3$ & 0.118 \\
\hline $\operatorname{EPAP}(\mathrm{cmH} 2 \mathrm{O})$, mean $\pm \mathrm{SD}$ & $7 \pm 2$ & $7 \pm 2$ & 0.980 \\
\hline IPPV on ICU admission, n (\%) & $7(36.8)$ & 39(61.9) & 0.054 \\
\hline $\mathrm{VT}(\mathrm{ml} / \mathrm{kg})$, mean $\pm \mathrm{SD}$ & $6.8 \pm 1.0$ & $7.1 \pm 1.2$ & 0.462 \\
\hline Pplat $(\mathrm{cmH} 2 \mathrm{O})$, mean $\pm \mathrm{SD}$ & $25 \pm 4$ & $24 \pm 6$ & 0.821 \\
\hline PEEP(cmH2O), mean $\pm S D$ & $10 \pm 4$ & $10 \pm 4$ & 1.000 \\
\hline $\mathrm{FiO} 2$, mean $\pm \mathrm{SD}$ & $0.74 \pm 0.15$ & $0.65 \pm 0.18$ & 0.066 \\
\hline Recruitment maneuvers, n (\%) & $6(35.3)$ & $17(30.9)$ & 0.735 \\
\hline NRM on ICU admission, n (\%) & $9(47.4)$ & 15(23.8) & 0.048 \\
\hline
\end{tabular}

Values are expressed as the mean \pm SD or Number (\%), unless otherwise indicated. $V T$ tidal volume of predicted body weight, Pplat plateau pressure, PEEP positive end-expiratory pressure, FiO2 fraction of inspired oxygen, IPAP inspiratory positive airway pressure, EPAP expiratory positive airway pressure 
clinicians and widespread implementation of PCR technique for PJP diagnosis might account for the discrepancy between Chen's study and ours.

\section{Limitation}

The main limitation of the current study was the retrospective nature of the investigation. Considering the relatively low incidence of PJP in ICU, it would be reasonable to prospectively collect data in the future investigation based on current finding. The second limitation was the small population recruited. To our best knowledge, only one study [8] included more ICU PJP patients than ours (88 vs. 82 ). However, because we focused on the prognostic factors for PJP in an ICU setting, our findings were more helpful to the ICU patients. Third, PJP was diagnosed on the basis of PJP PCR result in some patients. Due to the colonization of PJP, there is a possibility of false positive results. According to recent studies [32, 33], quantitative real-time Polymerase Chain Reaction (PCR) might be helpful in discriminating colonization from infection. However, those real-time PCR was not available in our centers during the study period. Nevertheless, all patients included in analysis had symptoms and their radiographic findings were compatible with PJP.

\section{Conclusion}

Our finding suggested that PJP in non-HIV patients requiring ICU admission remains a challenge for clinician. Poor prognostic factors included older age, lower WBC, and development of pneumomediastinum.

\section{Additional files}

Additional file 1: Table S2. PJP diagnosis by methenamine and PCR in participating centers. (DOCX $59 \mathrm{~kb}$ )

Additional file 2: Table S1. Summary of published studies. (DOCX $88 \mathrm{~kb}$ ) Additional file 3: Table S3. Comparison of patients screened for P.JP and patient enrollment in 3 studies in Peking Union Medical College Hospital. (DOCX 73 kb)

\section{Abbreviations}

APACHE: Acute physiology and chronic health evaluation;

BALF: Bronchoalveolar lavage fluid; CJFH: China-Japan Friendship Hospital; CMV: Cytomegalovirus; EORTC: European Organization for Research and Treatment of Cancer; FiO2: Fraction of inspired oxygen; HAART: Highly active antiretroviral therapy; ICU: Intensive care unit; PEEP: Positive end-expiratory pressure; PJP: Pneumocystis jirovecii pneumonia; PUMCH: Peking Union Medical College Hospital; TMP-SMX: Trimethoprim-sulfamethoxazole; VAP: Ventilator-associated pneumonia; WBC: White blood cell count

\section{Acknowledgements}

Not applicable.

\section{Availability of data and materials}

The data of this study will not be shared due to lack of ethical approval.

\section{Authors' contributions}

LW and BD had full access to the data and are responsible for the integrity of the data and final decision to submit. XH, LC, LQF, and WJ contributed to data collection; XH, XYH, JMP, CYW, and QYZ. contributed to the design of the study, data collection, data interpretation, and data analysis; and all authors contributed to the writing of the manuscript and have approved the final version for submission.

\section{Competing interests}

The authors declare that they have no competing interests. All authors have reported that no potential conflicts of interest exist with any companies/ organizations whose products or services may be discussed in this article. All authors declared no support from any organization for the submitted work.

\section{Ethics approval and consent to participate}

This study was approved by the institutional review board of Peking Union Medical College Hospital (S-K116). Due to the retrospective nature of the study, informed written consent was waived.

\section{Author details}

${ }^{1}$ Medical ICU, Peking Union Medical College Hospital, Peking Union Medical College and Chinese Academy of Medical Sciences, 1 Shuaifuyuan, Dongcheng district, Beijing 100730, China. ${ }^{2}$ Department of Respiratory and Critical Care Medicine, China-Japan Friendship Hospital, 2 Yinghua Dongjie, Hepingli, Beijing 100029, China. ${ }^{3}$ Department of General Internal Medicine, Shijingshan hospital, Capital Medical University, 24 Shijingshan street, Shijingshan district, Beijing 100043, China. ${ }^{4}$ Intensive Care Unit, Fifth Hospital of Shi Jia Zhuang, 42 Tanan street, Yuhua district, Shijiazhuang 050021, China.

Received: 21 July 2016 Accepted: 20 September 2016

Published online: 29 September 2016

\section{References}

1. Buchacz K, Baker RK, Palella Jr FJ, Chmiel JS, Lichtenstein KA, Novak RM, Wood KC, Brooks JT, Investigators H. AIDS-defining opportunistic illnesses in US patients, 1994-2007: a cohort study. AIDS. 2010;24(10):1549-59.

2. Powell K, Davis JL, Morris AM, Chi A, Bensley MR, Huang L. Survival for patients With HIV admitted to the ICU continues to improve in the current era of combination antiretroviral therapy. Chest. 2009;135(1):11-7.

3. Morris A, Norris KA. Colonization by Pneumocystis jirovecii and its role in disease. Clin Microbiol Rev. 2012:25(2):297-317.

4. Akgun KM, Pisani M, Crothers K. The changing epidemiology of HIV-infected patients in the intensive care unit. J Intensive Care Med. 2011;26(3):151-64.

5. Bienvenu A-L, Traore K, Plekhanova I, Bouchrik M, Bossard C, Picot S. Pneumocystis pneumonia suspected cases in 604 non-HIV and HIV patients. Int J Infect Dis. 2016;46:11-7.

6. Festic $\mathrm{E}$, Gajic $\mathrm{O}$, Limper AH, Aksamit TR. Acute respiratory failure due to pneumocystis pneumonia in patients without human immunodeficiency virus infection: outcome and associated features. Chest. 2005;128(2):573-9.

7. Hardak E, Neuberger A, Yigla M, Berger G, Finkelstein R, Sprecher H, Oren I. Outcome of Pneumocystis jirovecii pneumonia diagnosed by polymerase chain reaction in patients without human immunodeficiency virus infection. Respirology. 2012;17(4):681-6.

8. Kim SJ, Lee J, Cho Y-J, Park YS, Lee C-H, II Yoon H, Lee S-M, Yim J-J, Lee JH, Yoo C-G, et al. Prognostic factors of Pneumocystis jirovecii pneumonia in patients without HIV infection. J Infect. 2014;69(1):88-95.

9. Vogel MN, Vatlach M, Weissgerber P, Goeppert B, Claussen CD, Hetzel J, Horger M. HRCT-features of Pneumocystis jiroveci pneumonia and their evolution before and after treatment in non-HIV immunocompromised patients. Eur J Radiol. 2012;81(6):1315-20.

10. De Pauw B, Walsh TJ, Donnelly JP, Stevens DA, Edwards JE, Calandra T, Pappas PG, Maertens J, Lortholary O, Kauffman CA, et al. Revised definitions of invasive fungal disease from the European Organization for Research and Treatment of Cancer/Invasive Fungal Infections Cooperative Group and the National Institute of Allergy and Infectious Diseases Mycoses Study Group (EORTC/MSG) Consensus Group. Clin Infect Dis. 2008;46(12):1813-21.

11. Ljungman P, Griffiths P, Paya C. Definitions of cytomegalovirus infection and disease in transplant recipients. Clin Infect Dis. 2002;34(8):1094-7.

12. Lee WS, Hsueh PR, Hsieh TC, Chen FL, Ou TY, Jean SS. Caspofungin salvage therapy in Pneumocystis jirovecii pneumonia. J Microbiol Immunol Infect. 2016:3-4. In press. 
13. Ali HS, Hassan IF, George S. Extra corporeal membrane oxygenation to facilitate lung protective ventilation and prevent ventilator-induced lung injury in severe Pneumocystis pneumonia with pneumomediastinum: a case report and short literature review. BMC Pulm Med. 2016;16(1):52.

14. Cheng W-L, Ko W-C, Lee N-Y, Chang C-M, Lee C-C, Li M-C, Li C-W. Pneumomediastinum in patients with AIDS: a case report and literature review. Int J Infect Dis. 2014;22:31-4.

15. X-d M, Gao L, Wang R-g, Su L, Zhang C, Que C-I, Li H-C, Wang G-f. Retrospective analysis of relationship between radiological features and prognoses of pneumocystis pneumonia in non-AIDS immunocompromised patients. Zhonghua Yi Xue Za Zhi. 2012;92(38):2703-6.

16. Lemiale V, Debrumetz A, Delannoy A, Alberti C, Azoulay E. Adjunctive steroid in HIV-negative patients with severe Pneumocystis pneumonia. Respir Res. 2013;14(1):1.

17. Cho J-Y, Kim D-M, Kwon Y, Yoon S, Lee S. Newly formed cystic lesions for the development of pneumomediastinum in Pneumocystis jirovecii pneumonia. BMC Infect Dis. 2009;9(1):171.

18. Li M-C, Lee N-Y, Lee C-C, Lee H-C, Chang C-M, Ko W-C. Pneumocystis jiroveci pneumonia in immunocompromised patients: Delayed diagnosis and poor outcomes in non-HIV- infected individuals. J Microbiol Immunol Infect. 2014; 47(1):42-47.

19. Guo F, Chen Y, Yang S-L, Xia H, Li X-W, Tong Z-H. Pneumocystis pneumonia in HIV-infected and immunocompromised non-HIV infected patients: a retrospective study of two Centers in China. PLoS One. 2014;9(7):e101943.

20. Roembke F, Heinzow HS, Gosseling T, Heinecke A, Domagk D, Domschke W, Meister T. Clinical outcome and predictors of survival in patients with pneumocystis jirovecii pneumonia - results of a tertiary referral centre. Clin Respir J. 2013;8(1):86-92.

21. Boonsarngsuk $V$, Sirilak $S$, Kiatboonsri S. Acute respiratory failure due to Pneumocystis pneumonia: outcome and prognostic factors. Int J Infect Dis. 2009;13(1):59-66.

22. Monnet X, Vidal-Petiot E, Osman D, Hamzaoui O, Durrbach A, Goujard C, Miceli C, Bourée P, Richard C. Critical care management and outcome of severe Pneumocystis pneumonia in patients with and without HIV infection. Crit Care. 2008;12(1):R28.

23. Roblot F, Godet C, Le Moal G, Garo B, Faouzi Souala M, Dary M, De Gentile L, Gandji JA, Guimard Y, Lacroix C, et al. Analysis of underlying diseases and prognosis factors associated with Pneumocystis carinii pneumonia in immunocompromised HIV-negative patients. Eur J Clin Microbiol Infect Dis. 2002;21(7):523-31.

24. Mansharamani NG, Garland R, Delaney D, Koziel H. Management and outcome patterns for adult Pneumocystis carinii pneumonia, 1985 to 1995 : comparison of HIV-associated cases to other immunocompromised states. Chest. 2000;118(3):704-11.

25. Chen M, Tian X, Qin F, Zhou J, Liu J, Wang M, Xu K-F. Pneumocystis pneumonia in patients with autoimmune diseases: a retrospective study focused on clinical characteristics and prognostic factors related to death. PLoS One. 2015;10(9):e0139144

26. Roux A, Canet E, Valade S, Gangneux-Robert F, Hamane S, Lafabrie A, Maubon D, Debourgogne A, Le Gal S, Dalle F, et al. Pneumocystis jirovecii pneumonia in patients with or without AIDS, France. Emerg Infect Dis. 2014; 20(9):1490-7.

27. Zahar JR, Robin M, Azoulay E, Fieux F, Nitenberg G, Schlemmer B. Pneumocystis carinii pneumonia in critically ill patients with malignancy: a descriptive study. Clin Infect Dis. 2002;35(8):929-34.

28. Fillatre $P$, Chevrier $S$, Revest M, Gacouin A, Jouneau S, Leroy H, Robert-Gangneux $F$, Minjolle S, Le Tulzo Y, Tattevin P. Human herpes virus co-infection is associated with mortality in HIV-negative patients with Pneumocystis jirovecii pneumonia. Eur J Clin Microbiol Infect Dis. 2013;32(2):189-94.

29. Green H, Paul M, Vidal L, Leibovici L. Prophylaxis of pneumocystis pneumonia in immunocompromised non-HIV-infected patients: systematic review and meta-analysis of randomized controlled trials. Mayo Clin Proc. 2007:82(9):1052-9.

30. Moon SM, Kim T, Sung H, Kim M-N, Kim S-H, Choi S-H, Jeong J-Y, Woo JH, Kim YS, Lee S-O. Outcomes of moderate-to-severe Pneumocystis pneumonia treated with adjunctive steroid in non-HIV-infected patients. Antimicrob Agents Chemother. 2011;55(10):4613-8.

31. Stern A, Green H, Paul M, Vidal L, Leibovici L. Prophylaxis for Pneumocystis pneumonia (PCP) in non-HIV immunocompromised patients. Cochrane Database Syst Rev. 2014;10:CD005590.
32. Botterel F, Cabaret O, Foulet F, Cordonnier C, Costa JM, Bretagne S. Clinical significance of quantifying Pneumocystis jirovecii DNA by using real-time PCR in bronchoalveolar lavage fluid from immunocompromised patients. J Clin Microbiol. 2012;50(2):227-31.

33. Robert-Gangneux F, Belaz S, Revest M, Tattevin P, Jouneau S, Decaux O, Chevrier S, Le Tulzo Y, Gangneux JP. Diagnosis of Pneumocystis jirovecii pneumonia in immunocompromised patients by real-time PCR: a 4-year prospective study. J Clin Microbiol. 2014;52(9):3370-6.

\section{Submit your next manuscript to BioMed Central and we will help you at every step:}

- We accept pre-submission inquiries

- Our selector tool helps you to find the most relevant journal

- We provide round the clock customer support

- Convenient online submission

- Thorough peer review

- Inclusion in PubMed and all major indexing services

- Maximum visibility for your research

Submit your manuscript at www.biomedcentral.com/submit
Biomed Central 